\title{
Cross-species hybridisation of human and bovine orthologous genes on high density cDNA microarrays
} James Adjaye*1, Ralf Herwig ${ }^{1}$, Doris Herrmann², Wasco Wruck ${ }^{1}$, Alia BenKahla ${ }^{1}$, Thore C Brink ${ }^{1}$, Monika Nowak ${ }^{2}$, Joseph W Carnwath ${ }^{2}$, Claus Hultschig ${ }^{1}$, Heiner Niemann ${ }^{2}$ and Hans Lehrach ${ }^{1}$

\author{
Address: ${ }^{1}$ Max Planck Institute for Molecular Genetics, (Department of Vertebrate Genomics), Ihnestrasse 73, D-14195, Berlin, Germany and \\ 2Institute for Animal Science, (Department of Biotechnology), Mariensee, 31535 Neustadt, Germany \\ Email: James Adjaye* - adjaye@molgen.mpg.de; Ralf Herwig - herwig@molgen.mpg.de; Doris Herrmann - herrmann@tzv.fal.de; \\ Wasco Wruck - wruck_w@molgen.mpg.de; Alia BenKahla - kahla@molgen.mpg.de; Thore C Brink - brink@molgen.mpg.de; \\ Monika Nowak - nowak@tzv.fal.de; Joseph W Carnwath - carnwath@tzv.fal.de; Claus Hultschig - hultschig@molgen.mpg.de; \\ Heiner Niemann - niemann@tzv.fal.de; Hans Lehrach - lehrach@molgen.mpg.de \\ * Corresponding author
}

Published: 28 October 2004

BMC Genomics 2004, 5:83 doi:10.1186/147|-2164-5-83

This article is available from: http://www.biomedcentral.com/I47|-2/64/5/83

(C) 2004 Adjaye et al; licensee BioMed Central Ltd.

This is an Open Access article distributed under the terms of the Creative Commons Attribution License (http://creativecommons.org/licenses/by/2.0), which permits unrestricted use, distribution, and reproduction in any medium, provided the original work is properly cited.
Received: 23 June 2004

Accepted: 28 October 2004

\begin{abstract}
Background: Cross-species gene-expression comparison is a powerful tool for the discovery of evolutionarily conserved mechanisms and pathways of expression control. The usefulness of cDNA microarrays in this context is that broad areas of homology are compared and hybridization probes are sufficiently large that small inter-species differences in nucleotide sequence would not affect the analytical results. This comparative genomics approach would allow a common set of genes within a specific developmental, metabolic, or disease-related gene pathway to be evaluated in experimental models of human diseases. The objective of this study was to investigate the feasibility and reproducibility of cross-species analysis employing a human cDNA microarray as probe.
\end{abstract}

Results: As a proof of principle, total RNA derived from human and bovine fetal brains was used as a source of labelled targets for hybridisation onto a human cDNA microarray composed of 349 characterised genes. Each gene was spotted 20 times representing 6,980 data points thus enabling highly reproducible spot quantification. Employing high stringency hybridisation and washing conditions, followed by data analysis, revealed slight differences in the expression levels and reproducibility of the signals between the two species. We also assigned each of the genes into three expression level categories- i.e. high, medium and low. The correlation co-efficient of cross hybridisation between the orthologous genes was 0.94 . Verification of the array data by semiquantitative RT-PCR using common primer sequences enabled co-amplification of both human and bovine transcripts. Finally, we were able to assign gene names to previously uncharacterised bovine ESTs.

Conclusions: Results of our study demonstrate the harnessing and utilisation power of comparative genomics and prove the feasibility of using human microarrays to facilitate the identification of co-expressed orthologous genes in common tissues derived from different species. 


\section{Background}

Microarrays are routinely used for large scale transcriptome analyses and have been widely and successfully employed for simultaneously monitoring the expression of a potentially unlimited number of genes in parallel, thus providing the basis for identifying genes differentially expressed in distinct cell-types, developmental stages, disease states and cells subjected to exogenous reagents [1]. The rapid and significant improvements of cDNA-chip technologies and the availability of multi-species gene catalogues within the various data bases have made possible the comparison of gene expression levels within a single mammalian organism and across different organisms on a large-scale.

The advantages of cross-species hybridisation are twofold. First, cross-species gene-expression comparison is a powerful tool for the discovery of evolutionarily conserved mechanisms and pathways of expression control. The advantage of cDNA microarrays in this context is that broad areas of homology are compared and hybridization probes are sufficiently large so that small inter-species differences in nucleotide sequence would not affect the analytical results. This comparative genomics approach would allow a common set of genes within a specific developmental, metabolic, or disease-related gene pathway to be evaluated in experimental models of human diseases. Second, the use of microarrays in studies in mammalian species other than human and rodents, for example nonhuman primates, bovine, sheep and porcine may advance our understanding of human health and disease, for example the use of animal models in drug target validation. However, the inavailability of adequate sequence data and commercial cDNA and oligonucleotide microarrays keeps this technology beyond the reach of investigators working on economically and scientifically important large domestic species such as cattle, pigs and sheep. A potential solution to this problem is the use of cross-species hybridisations, i.e, human sequencebased arrays as tools for undertaking comparative genome expression studies. Such analyses have been performed using ape brain RNA as target on a human oligonucleotide array [2] and pig, mouse and Atlantic salmon RNA on human nylon arrays- [3-7]. These types of studies represent critical areas of research directly related to the understanding of human diseases because nonhuman primates, bovine, sheep and porcine play a crucial role in biomedicine, such as, organ transplantation, vaccine development, viral pathogenesis, gene therapy and a host of other human health-related technologies.

A crucial step employing domestic animals in biomedicine is genetic modification which requires extensive embryo and embryo-related technologies, such as in vitro production of embryos for stem cell derivation and somatic nuclear transfer cloning. Employing the bovine model and sensitive RT-PCR assays, it has been shown that the majority of embryos derived from such sources display distinct mRNA expression patterns in a variety of developmentally important genes compared to their in vivo derived counterparts [8]. Some of these aberrations lead to "Large offspring syndrome", a complex of multiple pathologies observed in offspring derived from in vitro production and/or nuclear transfer of which significant oversize is a predominant feature [9]. Analysis of mRNA expression patterns in early embryos via cDNA microarray technology would provide insights into the function of gene regulatory networks and would thus be a major step forward in unravelling molecular mechanisms underlying developmental abnormalities. The technology to amplify the minute amounts of mRNA in early embryos without significantly altering the ratio of the various mRNAs in the original cell has recently been described $[10,11]$ and a prototype mouse cDNA-macroarray enriched in embryonic sequences has been developed [11].

Important criteria for evaluating any microarray system include the reproducibility of the data generated, the specificity of detection of the targeted gene, and the validity of the results that identify and establish differential gene expression. The experiments described here show the systematic validation of cross-species microarray analysis, with emphasis on the reproducibility and statistical analysis of generated data using standard microarray data analysis tools.

Specifically, we investigated the feasibility and reproducibility of cross-species hybridisation of orthologous genes within a defined developmental and metabolic pathway using as a test case and the first description of its kind, human and bovine fetal brain RNA as Cy-dye labelled targets on a human cDNA microarray. The microarray is composed of 349 genes each spotted 20 times to ensure reproducible validation by independent technologies such as semi-quantitative RT-PCR as carried out in this study, or alternatively, Real-Time PCR and Northern blot analysis.

\section{Results \\ Array fabrication and gene annotation}

The human cDNA microarray used in this study consisted of 349 fully sequenced and annotated cDNAs as described in the Supplemental Table 1. Thirty-five spots containing only spotting solution ( $3 x$ SSC / $1.5 \mathrm{M}$ betaine) served as negative controls. In addition, in a separate control plate, the housekeeping genes HPRT and $\beta$-ACTIN (made up of a dilution series of 25-, 50-, 100-, 150- and $200 \mathrm{ng} / \mu \mathrm{l}$ ) were employed as endogenous guide dots to enable accurate grid placement prior to image analysis. These spots can be seen as intense yellow signals at the periphery of 
Table I: Summary of primer sequences, annealing temperatures and size of amplicons. Derivation of primer sequences: $H$ and $B$ denote human and bovine respectively. The nucleotide positions of the primer sequences are in parenthesis. Nucleotides highlighted in bold denote differences in the orthologous gene sequences.

\begin{tabular}{|c|c|c|c|c|}
\hline Genes & Primer sequences $\left(5^{\prime}-3^{\prime}\right)$ & Annealing temperature $\left({ }^{\circ} \mathrm{C}\right)$ & Fragment size (bp) & Accession numbers \\
\hline $\mathrm{CDC} 27$ & $\begin{array}{l}(I 226-1250) \mathrm{H} \\
\text { TATTACATCTCCCCCAAACGCACTG } \\
(1512-1536) \mathrm{H} \\
\text { CCATTTCACGAAGAAGGCTCATCAA }\end{array}$ & 54 & 311 & $\begin{array}{l}\text { NM_00I256 H } \\
\text { CBI70694 B }\end{array}$ \\
\hline CDC6 & $\begin{array}{l}(89-113) \text { B } \\
\text { TCCCAAGCGGGTTGGTGTTATTCAC } \\
(272-296) \text { B } \\
\text { GCGACAGACTGTACTGTAGGCTTCA }\end{array}$ & 54 & 208 & $\begin{array}{l}\text { NM_00I254 H } \\
\text { BF600055 B }\end{array}$ \\
\hline PCNA & $\begin{array}{l}(195-217)^{\text {B }} \\
\text { GAGGCGCTTAAGGATCTCATCAA } \\
(554-576)^{\text {в }} \\
\text { ATTCACCAGAAGGCATCTTTACT }\end{array}$ & 54 & 382 & $\begin{array}{l}\text { AF527838 H } \\
\text { CB53I5I9 B }\end{array}$ \\
\hline $\mathrm{SLCIIA3}$ & $\begin{array}{l}(38-61)^{\text {в }} \\
\text { ACCCCTGGAGGGAACTCATCTAAT } \\
(290-313)^{\text {в }} \\
\text { GCTGATGCTCCCATCAAAATACTG }\end{array}$ & 57 & 276 & $\begin{array}{l}\text { AF2I5636.1 H } \\
\text { BG6897/2 B }\end{array}$ \\
\hline VDAC2 & $\begin{array}{l}(652-675) \text { в } \\
\text { CCTCGGTTGTGATGTTGACTTTGA } \\
(1076-1099) \text { в } \\
\text { GTGGCCTCCAGCATTAATGCTCTT }\end{array}$ & 57 & 448 & $\begin{array}{l}\text { NM_003375.I H } \\
\text { TCI528II B }\end{array}$ \\
\hline CALNEXIN & $\begin{array}{l}\text { (I054-I078): H } \\
\text { GCTGGTTAGATGATGAGCCTGAGTA } \\
\text { (I 266-I290): H } \\
\text { TCCTGGGTTTCCAGATTCCCTGGTA }\end{array}$ & 56 & 237 & $\begin{array}{l}\text { NM_001746.1 H } \\
\text { BM431III B }\end{array}$ \\
\hline$\beta$-ACTIN & $\begin{array}{l}44 I-46 I) \mathrm{H} \\
\text { GTTGCTATCCAGGCTGTGCT } \\
(890-9 I 0) \mathrm{H} \\
\text { CGGATGTCCACGTCACACTT }\end{array}$ & 60 & 469 & $\begin{array}{l}\text { NM_00II I0I H } \\
\text { NM_I } 173979 \text { B }\end{array}$ \\
\hline
\end{tabular}

each block as portrayed in Figure 1A. The embryonic-specific gene, OCT-4 and Arabidopsis cDNAs (Cab- T97312; Cwlp- T02614; Lhb1B1- T21965; Ohp- T22679) were included as negative controls to monitor hybridisationspecificity. The transcription factor OCT-4 is expressed in human embryonic stem cells and primordial germ cells and is down-regulated upon differentiation $[10,12,13]$.

Classification of the genes according to Gene Ontology annotations (molecular function) and chromosomal location (Figures $1 \mathrm{~B}$ and $1 \mathrm{C}$ ) demonstrates that the selected genes encompass a range of twelve different functional classes and are located on all except the Y-chromosome. This implies that there is no obvious bias towards biological characteristics and the selected gene set can be viewed as representative for the current study.

\section{Global data characteristics}

Our experimental design incorporated dye-swaps and four replicated hybridisations. Three hundred and forty nine cDNAs, each representing a unique gene were spotted 20 times on each microarray. Four independent experiments were performed with human and bovine brain, respectively. Within each experiment the 20 replicates were averaged to yield a reliable signal for the respective probe. In the next step, the replicated signals from the different experiments were averaged to compute overall characteristics. The overall correlation of expression of the human and bovine genes is shown in Figure 2A. The regression line bears a slope close to one (1.13) thus indicating similar expression of human and bovine genes across the replicated experiments with a slight increase of expression in the human brain. The overall correlation coefficient is 0.94 .

Additionally, we computed MA-plots [14] in order to detect artificial dependencies of the log-ratio across the signal range. Figure $2 \mathrm{~B}$ illustrates a typical result from a specific hybridisation experiment. The horizontal lines mark the two-fold levels of over-expression in bovine and human, respectively. A clear observation is that a few of the 349 genes under investigation fall outside these thresholds with the expression of 5 genes being more than 2 -fold over-expressed in bovine and 16 genes more than 2-fold over-expressed in human. However, there is no 


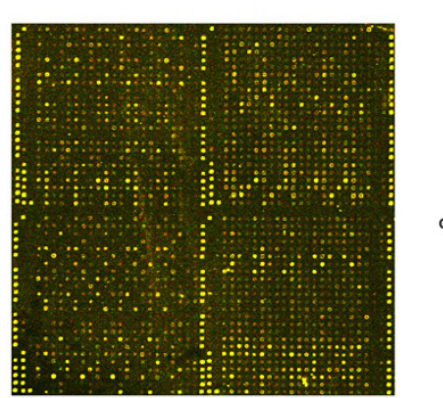

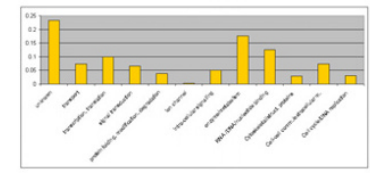

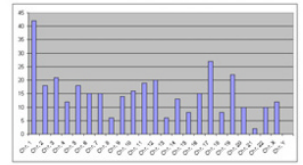

\section{Figure I}

Array fabrication and gene annotation (A) False-colour image generated from a bovine-Cy5, human-Cy3 hybridisation. False colour images were generated using the programme ScanAlyze, version 2.44 http://www.rana.lbl.gov/ EisenSoftware.htm. The full array $(24 \times 25$ spotting pattern) consists of 16 blocks with each gene spotted 5 times per block therefore 80 potential data points present for expression analysis per gene. A blow-up of 4 blocks illustrating the Chip design is presented. The ACTIN cDNAs acting as guidedots can be seen as intense yellow spots demarcating each block. In addition, the majority of spots appear yellow due to similar expression levels of the orthologous genes. (B) Functional annotation of the 349 genes as set out by the Gene Ontology Consortium http://rana.lbl.gov/EisenSoft ware.htm. The proportion of these genes within each functional / biological annotation is represented on the $Y$-axis and the annotation on the $X$-axis. (C) Chromosomal distribution of the 349 genes. The number of genes within each chromosome is represented on the $\mathrm{Y}$-axis and the chromosome numbers on the $\mathrm{X}$-axis.

increase or decrease in fold-change for the vast majority of genes and this is fairly stable across the signal range.

In order to measure whether a given gene was significantly expressed, we compared its cDNA's signal to a signal distribution derived from negative controls represented by approx. 2,500 empty spot positions on the array. After quantification of each array a low non-zero intensity is assigned to each of these empty spots reflecting the amount of background signal on the array. Since these positions are spread uniformly over the array, the distribution of these signals reflects the distribution for signal noise and is an indicator whether signals are at the background level or reflect reliable expression levels. For each cDNA we counted the relative proportion of empty positions on the array that are smaller than the actual observed intensity (BG-tag). BG-tags from replicated experiments for the same cDNA were averaged. Thus, high
A

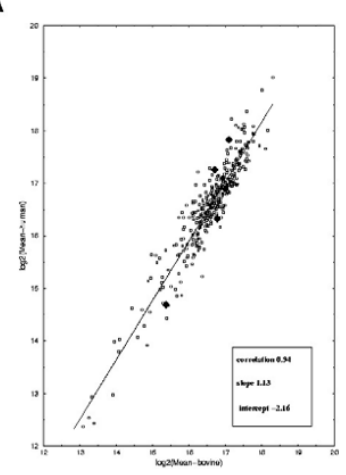

C

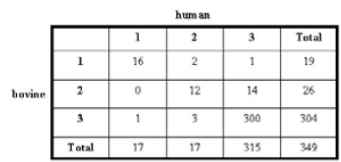

B

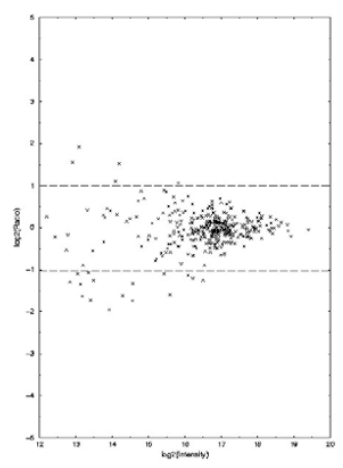

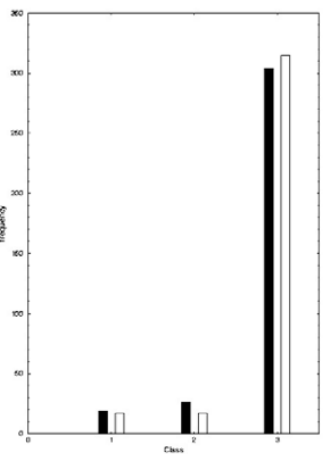

Figure 2

Global data characteristics (A) Global correlation of bovine (X-axis) and human ( $\mathrm{Y}$-axis) experiments. The plot shows a log-log (base 2) plot of the mean signal intensities from the four independent experiments for each gene. The red line shows the regression line, the box displays the parameters of the regression line (intercept, slope) and the overall correlation. Four genes that are significantly differentially expressed as judged by using the Wilcoxon's rank-sum test are denoted by black diamond shapes. (B) MA-plot of a single experiment. The $X$-axis show the log (base 2 ) of the squared product of Cy5-red and Cy3-green intensities of each gene in the same experiment. $Y$-axis shows the log-ratio (base 2) of red and green intensity. The horizontal lines mark the two-fold overexpressed genes (Top line; bovine over-expression and bottom line; human over-expression). (C). Classification of detection levels of the 349 genes. The genes were subdivided into three classes of expression levels. Class I (BGtag < 0.8) - low (not detected). Class 2 (BG-tag between 0.8 and 0.9) - medium (boarderline detection) and Class 3 (BGtag > 0.9) - high expression (readily detectable) The number of genes (X-axis) within each class ( $Y$-axis) is also shown in the figure (black $=$ bovine, white $=$ human).

values (close to one) indicate that the cDNA is expressed in the respective tissue whereas low values reflect noise. 
A
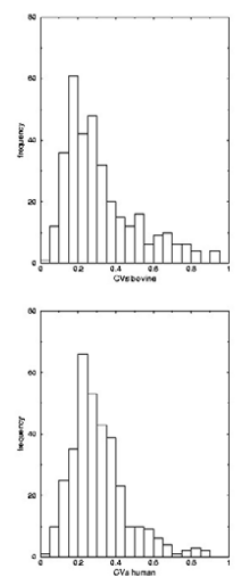

B

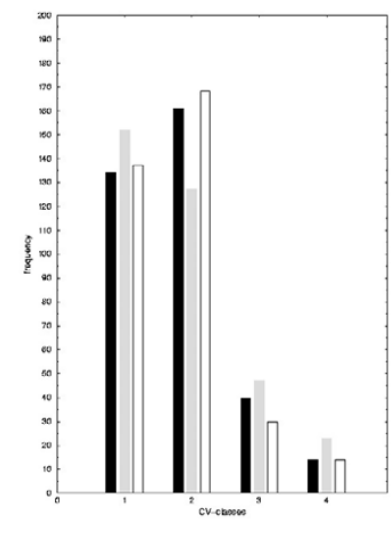

Figure 3

Data reproducibility and species variability (A) Histograms of CVs for human and bovine hybridisations. (B) Classification of signal variability in measured gene expression intensities. Human (white), bovine (grey) and mixed samples (black). CVs (co-efficient of variation) were calculated for repeated signal intensities of the four independent hybridisations and then sub-divided into four classes: Class I - CV < 0.25 high reproducibility, Class $2-\mathrm{CV}[0.25-0.5]$ good reproducibility, Class $3-\mathrm{CV}[0.5-0.75]$ medium reproducibility Class $4-C V>0.75$ poor reproducibility.

The limit of visual detection of a spot corresponds to a BGtag level of 0.9 , however there is a grey zone around this value. Comparison with RT-PCR analyses showed [15] that this level is consistent with the limit of detection at the $25^{\text {th }}$ cycle of a standard PCR reaction. cDNAs were considered as "detected" when their average BG-tag was above 0.9.

We grouped the 349 genes into three classes of expression levels with respect to their intensity values above background levels (i.e, BG-tag) with class 1 as low (notdetected), class 2 as medium (possibly detected) and class 3 as high level of expression (detected), respectively. Within class 1 , are 19 bovine and 17 human genes. Class 2 , comprises 26 bovine and 17 human genes and finally class 3 has 304 bovine and 315 human genes, respectively. The genes within expression class 1 have signal intensities below the detection level of our microarray analysis platform and as such these genes can be designated as "Absent" (See Figure 2C for a graphical illustration). A list of all the intensity values is given (see Additional file 1).
A

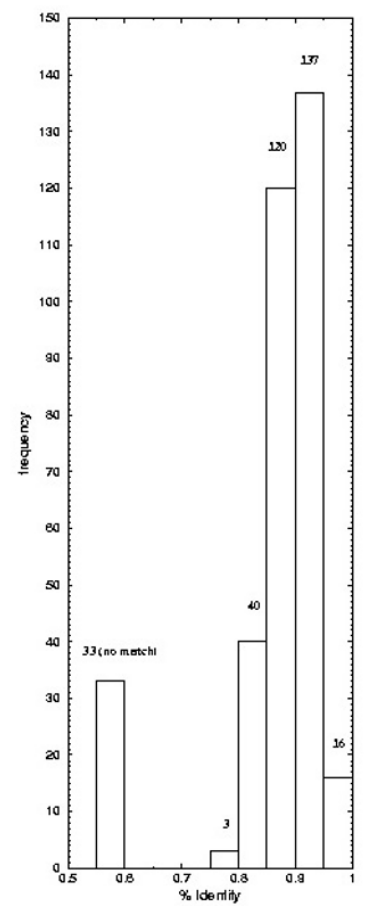

B

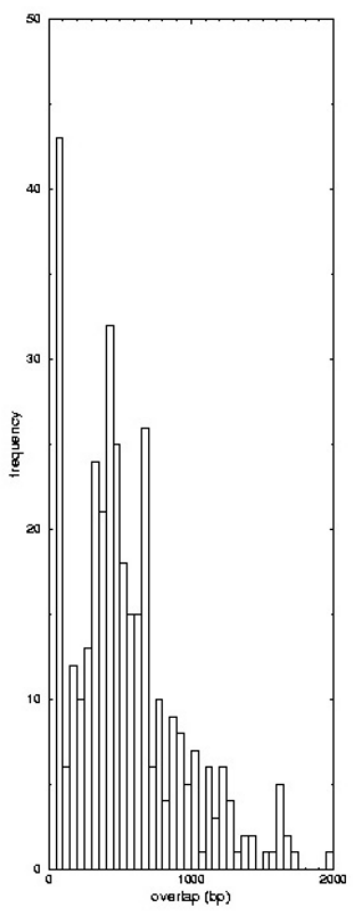

Figure 4

Nucleotide sequence alignments of bovine and human transcripts All 349 human genes were matched against the TIGR Bos taurus gene index, BtGI Release 8.0, which contains 87,257 unique sequences. (A) Histogram of $\%$-identities with the best match. (B) Histogram of bp overlap with the best match.

\section{Data reproducibility and species variability}

An essential criterion for the applicability of cross-species experiments is the data reproducibility. For example, if human probes hybridise to bovine mRNA with far less reproducibility than to human mRNA high changes in expression of bovine tissues can be observed that are solely due to technical variability. In order to test whether the variability in gene expression levels is conserved within both species or in contrast, is higher in the bovine than in the human hybridisations, we calculated the coefficient of variation (CV) across the replicated experiments. Histograms are shown in Figure 3A. We then defined four classes of CVs for the genes (highly reproducible signals $\mathrm{CV}<0.25$, good reproducibility $-0.25<\mathrm{CV}<0.5$, medium reproducibility $-0.5<\mathrm{CV}<0.75$ and poor reproducibility $\mathrm{CV}>0.75$ ). Note that a $\mathrm{CV}$ of 1.0 indicates that the signal standard deviation is in the order of the signal 
A

$\begin{array}{llll} & \underline{\text { CDC27 }} & \text { CDC6 } & \text { PCNA } \\ \text { Cell cycle } & - & -\end{array}$

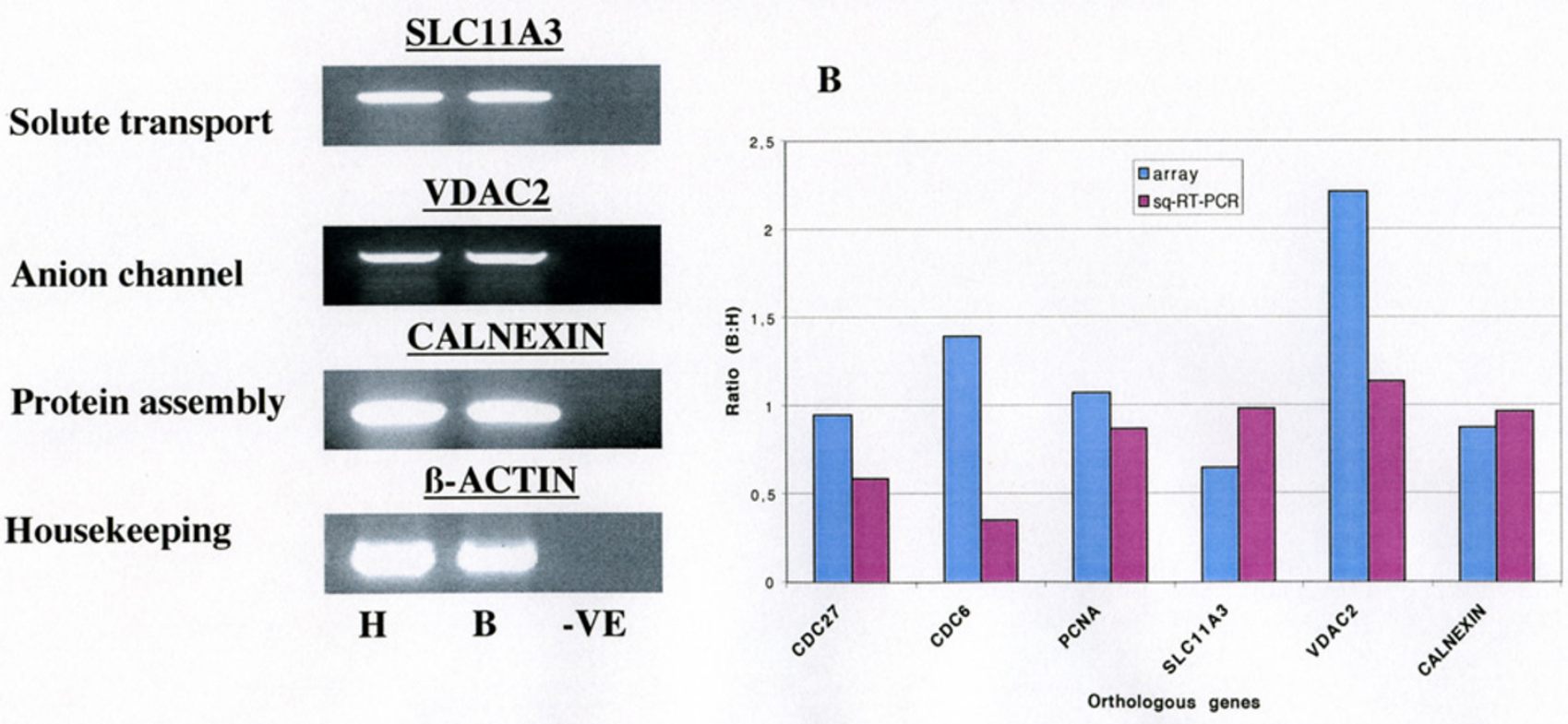

Figure 5

Independent verification of array results by semi-quantitative RT-PCR (A) Ethidium bromide stained gel illustrating independent verification of differential gene expression by semi-quantitative RT-PCR. PCR reactions were carried out in $50 \mu \mathrm{l}$ volumes, loaded in the order H- (human), B- (bovine) and VE- (water as negative control) then resolved on a $2 \%$ agarose gel as described in Materials and Methods. Each panel consist of genes of the same functional category - listed on the left hand side. A 100 bp ladder (Invitrogen) was used to confirm the sizes (Table I) of the PCR products. (B) A graphical illustration of the comparison of the expression levels of the orthologous genes as deduced by the microarray analysis and semiquantitative RTPCR.

itself and therefore no meaningful statement on the measurement can be made.

Figure 3B depicts the number of genes that fall within the respective CV-classes when analysing all experiments (human and bovine- black), human only (white) and bovine only (grey). There is a $10 \%$ decrease in the number of reproducible genes when comparing human and bovine but the overall effect is similar. Approximately 305 genes show a $\mathrm{CV}<0.5$ with human brain (87\%) compared to 280 with bovine brain (80\%). Only 15 genes $(4 \%)$ show high variability across the experiments for human brain compared to 20 genes $(5.7 \%)$ for bovine brain. Figure $3 \mathrm{~B}$ illustrates that there is a slight but not dramatic increase in variability when performing cross-species hybridisation (the number of variable genes (class 3 and 4 ) increase by a factor of 1.5). However, for the vast majority of the genes the reproducibility is similar. The observed effect is not due to exact cutoff values set for the different CV-classes since a slight shifting of the class borders leads to the similar results. For example, shifting the borders about a slight factor of $\vartheta= \pm 0.05$ to the left/right respectively gives approximately the same factor of increase for variable bovine to variable human genes. 


\section{Differential gene expression in bovine and human fetal brain}

Three statistical tests that judge the significance of differences in the levels of gene expression in human and bovine fetal brains were employed (Student's t-test, Welch-test and, Wilcoxon's rank-sum test) as described previously [16]. Tests were carried out with the four independent experiments in bovine and human brain, respectively. Note that with independent experiments we mean the independent technical replicates since we do not employ different biological replicates in our study. Messenger RNA levels of seven genes were significantly ( $\mathrm{p}<$ 0.05 with Student's t-test and Welch test) different between the two species, whilst four genes were found to be differentially expressed only with the Wilcoxon test ( $p$ $<0.05)$. This emphasizes that the Wilcoxon test is more conservative than the parametric tests. In contrast to the two parametric tests that assume a specific parametric signal distribution (Gaussian distribution) for the underlying signal series, the Wilcoxon test is non-parametric. Thus, P-values calculated by this test are valid in a more general set-up, i.e. for larger classes of probability distributions, than with the other tests. Furthermore, since the Wilcoxon test is based on ranks rather than on the underlying signals it is a more robust procedure in the sense that it is less sensitive against outliers from the model assumptions. These four genes are, ZNF278, APOARGC, KIAA1609 and MGC12904 - highlighted as diamond shapes in Figure 2A. The expression levels of the vast majority $(98 \%)$ of the genes remained unchanged.

Furthermore, taking into account corrections for multiple testing, no gene is differentially expressed at the global experiment significance level of 0.05. For example, the lowest P-value of the Welch test is 1.98e-03, (APOARGC). Thus, Holm's setp-wise correction would start with an adjusted experiment level P-value of $\mathrm{p}=0.05 / 349=1.43 \mathrm{e}$ 04. Similarly, measuring the false discovery rate by qvalues [17] results in no significant differential gene expression. Thus, we conclude that the level of expression of the individual 349 genes under investigation within human and bovine brain is roughly the same.

\section{Nucleotide sequence alignments of bovine and human transcripts}

In order to characterise some of the vast number of unknown bovine ESTs within the various databases we screened for orthologs to the human genes [18]. The 349 known human genes were screened against the TIGR Bos Taurus gene index (BtGI Release 8.0). Using high-quality matches ( $>85 \%$ identity, $>100$ bp overlap, E-value $<1.0 e-$ $15)$ we were able to ascertain the expression of 316 orthologous genes (see Additional file 1). Figure 4 shows the quality of the matches. Of these genes, 16 had sequence identities of greater than 95\%, 137 genes with identities between $90 \%$ and 95\%, 120 genes with identities of $85 \%$ to $90 \%$. The remaining genes did not meet the criterion for the assignment as orthologs [18]. Forty genes had identities of $80 \%$ to $85 \%$, 3 genes had identities between $75 \%$ and $80 \%$ and finally 33 genes did not have a significant BLAST hit. These matches are considered to be insignificant and therefore implying that these 33 human genes (assigned values (0) in Additional file 1) do not as yet have their bovine homologs present in the current bovine databases.

Employing a comparative genomics approach we have functionally annotated previously uncharacterised bovine ESTs. These genes are depicted in the Supplemental Table as bovine ESTs lacking a gene name or description in the TIGR Bos Taurus gene index. As an example, the gene SLC11A3 which encodes a protein which functions as a solute carrier has $92 \%$ nucleotide sequence identity with an overlap of 500 bp with its bovine orthologue. Additionally, we have demonstrated co-amplification of this transcript in both human and bovine fetal brain RNA using primers derived from the bovine sequence - Figure 5A and Table 1.

\section{Verification of expression levels of cross-hybridised orthologous genes by semi-quantitative RT-PCR}

Semi-quantitative RT-PCR was used to confirm the deduced expression data generated by the high stringency cross-species hybridisation. We selected a set of genes belonging to distinct families based on their published functional annotation, for example, cell cycle (CDC27, CDC6 and PCNA), solute transport- (SLC11A3), protein assembly- (CALNEXIN), anion channel- (VDAC2) and as an endogenous reference, the housekeeping gene, $\beta$ ACTIN. Primer sequences were designed to co-amplify the orthologous genes (Table 1).

The RT-PCR analyses (Figure 5A) using a common set of gene-specific primers clearly demonstrate co-amplifcation of the orthologous transcripts and, in addition, differences in expression levels between genes within the same species are discernable. For example, the house keeping gene $\beta$-ACTIN and CALNEXIN which is involved in protein assembly are more abundant than for examples genes involved in cell cycle control. Most importantly, the trends in the deduced ratios (see Additional file 1 and Figure 5B) of expression levels of orthologous genes from the hybridisation analysis are confirmed by the RT-PCR assay.

Discrepancies in the bovine-human ratio with array data and the semi-quantitative RT-PCR data are also observable. Furthermore, some of the genes (for example CDC6 and VDAC2) are expressed at a low level (expression class 1 , compare Figure 2C). Here, microarray measurements 
are less reliable than PCR-based measurements and one comes close to the borderline of the detection limit of microarrays.

\section{Discussion}

Customised and focussed microarrays containing orthologous genes related to function, tissue, or pathways are becoming widely adopted for studying mRNA expression patterns. In addition, the level of cross hybridisation between genes with high sequence identity is also of interest because arrays are not always available for mammalian species other than human and rodents so crossspecies hybridisations are often carried out $[19,20]$. In view of this, it is crucial to know whether the hybridisation conditions (i.e. the stringency used for the hybridisation and subsequent washes) would enable identification of altered gene expression across species. Here we have demonstrated cross-hybridisation of orthologous transcripts by adopting a high stringency hybridisation and wash protocol.

The overall correlation co-efficient of gene expression in the fetal brains of human and bovine was 0.94, with the regression line at a slope of 1.13 (Figure 2A). This suggests that the 349 genes under investigation have rather similar expression levels as judged by the intensity values of the human and bovine genes across the replicated experiments. The use of replicate experiments is clearly essential in order to ascertain true expression change. For example, judging single experiments we find a couple of genes escaping the 2-fold bounds. The MA-plot [14] shown in Figure $2 \mathrm{~B}$ suggests that of the 349 genes, 5 are overexpressed more than 2-fold in bovine and 16 are more than 2-fold over-expressed in human. However, most of the changes are due to experimental variability of this specific experiment.

An extremely important aspect of cross-species hybridisations is data reproducibility. A poor hybridisation experiment would lead to a high variability in the respective replicate experiments in human and bovine. With regards to this aspect, we identified fifteen genes (4\%) in human and twenty genes (5.7\%) in bovine with variability in expression levels in the four hybridisation experiments. In addition, approximately 305 genes have a CV of less than 0.5 with human brain $(87 \%)$ compared to 280 with bovine brain $(80 \%)$. However, this level of variability is low and can be expected due to the fact that the probe set used are of human origin. Moreover, the slight decrease in reproducibility due to species and nucleotide sequence differences can be compensated for by increasing the number of independent repetitions (biological and technical replicates) of the experiments. The unexpectedly low level of variability in gene expression levels between human and bovine fetal brain can also be attributed to the
80 data points examined per gene and the four replicate hybridisations (technical replicates) carried out for each species. Our finding further emphasises the need for sufficient technical and biological replicates in all microarray experiments.

In assessing differential gene expression in bovine and human fetal brain, we observed that the expression levels of the individual orthologous genes were roughly in the same broad range. For example, if one considers genes involved in pathways of cell cycle control, the microarray deduced ratios (bovine:human) were for CDC27: 0.95, CDC6: 1.36, PCNA: 1.07, respectively, whereas the deduced ratios derived from the semi-quantitative RTPCR assay were 0.59 for $C D C 27,0.35$ for $C D C 6$ and 0.87 for PCNA, respectively.

Similarly the comparative ratios for SLC11A3 and CALNEXIN are 0.65 vs. 0.98 and 0.88 vs. 0.97 . An illustration of co-amplification with common primers and also the confirmation of the array data for a selection of genes can be seen in Figures 5A and 5B. The expression ratios of all the other genes under investigation are given (see Additional file 1). Though co-amplification is possible by semi-quantitative RT-PCR, there is amplification bias related to primer mismatch (See Table 1). This effect was further highlighted when primer efficiencies were calculated using comparative Real-Time PCR (data not included). An important difference between the two techniques is that cDNA array hybridisation is less sensitive to minor mismatches than PCR primer annealing. The bias in amplification specificity is not a drawback in this study and species specific primers would obviously be used to compare gene expression levels in an experiment in which a developmental/metabolic pathway or disease model is investigated.

The similar expression ratio of the orthologous genes implicated in cell cycle control and initiation of eukaryotic genome replication which is conserved in all eukaryotes highlights the feasibility and importance of our study in examining conserved pathways operative within the fetal brains of human and bovine and of course across other species using human cDNA microarrays. A similar finding has also been confirmed when comparing yeast co-regulated genes against the archaeal and bacterial operons. This implies that the components of the protein translation process are conserved across organisms at the expression level with minor specific differences [21].

We also identified four significantly differentially expressed genes as judged by the Wilcoxon test. The Wilcoxon test is known to be more conservative than the Student's t-test and the Welch-test, however, we have more confidence in this test since it is distribution-free, in par- 
ticular it does not depend on the partly unrealistic assumption of an underlying Gaussian distribution.

The genes ZNF278 and APOARGC were 1.45 - and 1.66 fold over-expressed in human whereas KIAA1609 and MGC12904 were 1.60 - and 1.37-fold over-expressed in bovine. ZNF278 encodes a zinc finger-containing transcription factor that acts as a transcriptional repressor and also implicated in small round cell tumours [22]. The gene APOARGC encodes a protein with hydrolase activity. MGC12904 and KIAA1609 are uncharacterised ESTs so comments cannot be made with regard to their function.

Orthologous genes between human and mouse and between human and rat both have a mean of approximately $85 \%$ sequence identity $[18,23]$. In two independent and unrelated studies carried out on CDNA and 50mer oligonucleotide microarrays, cross-hybridisation was only observed with genes with $70 \%-80 \%$ and $50 \%-75 \%$ overall sequence identity, respectively $[24,25]$. With respect to these studies, our data unequivocally confirm the feasibility and reproducibility of cross-species hybridisation of orthologous genes within defined developmental and metabolic pathway(s) operative in human and bovine fetal brains. In addition, we have been able to assign gene names to previously uncharacterised bovine ESTs, thus, highlighting the importance of comparative genomics in identifying orthologous genes across species.

Furthermore, using this protocol of cross-species hybridisation, we have compared gene expression in bovine unfertilised oocytes and blastocyst using a larger microarray (The Human Ensembl Chip) comprising 15,500 fully sequenced and annotated genes and ESTs. The correlation co-efficient between the RNA samples is 0.26 , thus reflecting the diversity between oocyte derived maternal transcripts and embryonic transcripts derived from the blastocyst (Adjaye et al., unpublished).

\section{Conclusions}

In summary, this study highlights the significance and utilisation power of comparative genomics and also demonstrates the feasibility of using human cDNA microarrays to facilitate the identification of differentially expressed genes in human and bovine fetal brain. Our results indicate that cross-species hybridisation is not only a useful short-term solution for studying species for which gene maps, cDNA or oligo microarrays are not yet available, but also possesses tremendous power in enabling the unravelling of common evolutionary evolved mechanisms in different species.

\section{Methods}

\section{Microarray fabrication}

For the generation of probes for spotting, cDNA inserts from a single 384-well plate of non-redundant, fully sequenced, annotated human cDNA collection (Human Ensembl set RZPD1.1) were employed- (BenKahla et al., unpublished) and a second partial plate consisting of control genes and empty wells to be used for data normalisation were amplified by PCR in a 384-well format. Bacterial clones were transferred to the PCR plates using 384-well replicators (Genetix Ltd, New Hampshire, UK). PCR amplifications were carried out in a total volume of $25 \mu \mathrm{l}$ consisting of $1 \mathrm{x}$ PCR buffer, 1.5 M Betaine (Sigma, Germany), 1U Taq polymerase, $200 \mathrm{mM}$ of each dNTP (Invitek, Germany), 25 pmoles of M13-forward (5'GTAAAACGACGGCCA3') and M13-reverse (5'CAGGAAACAGCTATGAC3') primers respectively. Cycling parameters typically consisted of an initial denaturation step at $95^{\circ} \mathrm{C}$ for 5 min followed by 35 cycles of denaturation at $95^{\circ} \mathrm{C}$ for $1 \mathrm{~min}$, annealing at $55^{\circ} \mathrm{C}$ for 1 min, elongation at $72^{\circ} \mathrm{C}$ for $1 \mathrm{~min}$, then a final elongation step at $72^{\circ} \mathrm{C}$ for $10 \mathrm{~min}$. All amplified products were analysed by agarose gel electrophoresis and in all cases single bands were obtained per gene.

Purification was carried out using isopropanol precipitations. In brief, $24 \mu \mathrm{l}$ of the PCR product was transferred into 384-well Genetix plates (Genetix Ltd, New Hampshire, UK) previously filled with $18 \mu \mathrm{l}$ of isopropanol per well. The samples were mixed by gentle vortexing after sealing the plates with transparent tape. After precipitation overnight at $-20^{\circ} \mathrm{C}$, the plates were spun at $3,500 \mathrm{rpm}$ for $2 \mathrm{hrs}$ at $21^{\circ} \mathrm{C}$, the isopropanol decanted off and the plates dried briefly in a SpeedVac without heating. The pellets were resuspended in $6 \mu$ of spotting solution (3x SSC /1.5 M Betaine (N, N, N-trimethylglycine; Sigma, Germany)). Random sampling of cDNAs revealed concentrations ranging between 200 to $300 \mathrm{ng} / \mu \mathrm{l}$. Finally the samples in each well were spotted twenty times (for signal averaging) with four slit pins arranged in a $2 \mathrm{x} 2 \mathrm{y}$ printhead (Chipmaker 2 pins, Telechem, Sunnyvale, CA, USA). Prior to every spotting run the performance of all components of the robot is carefully tested - in particular the performance of the washing station in test runs designed to detect possible sample carry over. A modified Genetix Qarray (Genetix Ltd, New Hampshire, UK), controlled by a novel in-house software was used for the arraying of the samples on SuperAmine ${ }^{\mathrm{TM}}$ aminosilane-coated microscope slides (Telechem, Sunnyvale, Ca, USA). Postprocessing of the arrays was performed using 1,2-dichloroethane and the acylating catalyst $\mathrm{N}$-methylimidazole following protocols described previously [26]. 


\section{Isolation of total RNA}

Human brain RNA was isolated from a medically terminated fetus at 10 weeks gestation. This was provided by the MRC-funded Human Embryonic Tissue Bank maintained at the Institute of Child Health and University College London, England. Bovine fetal brain was obtained from a 3-4 months old bovine fetus at a local abattoir near Mariensee, Germany. Immediately after slaughter of the pregnant female, the tissue was plunged into liquid nitrogen and transported to the laboratory. Brain tissues were homogenised using a Dounce homogeniser, total RNA isolated using TRIzol reagent (Invitrogen) and further purified using phenol/chloroform extractions and precipitation with ethanol after DNase 1 (Promega) treatment. All procedures were as described by the manufacturers. RNA Purity, integrity and concentrations were evaluated on the Agilent 2100 Bioanalyzer. High quality RNAs with A260/A280 ratio over 1.8 with intact ribosomal $28 \mathrm{~S}$ and $18 \mathrm{~S}$ RNA bands were utilised for subsequent labelling reactions.

\section{Direct labelling of RNA and hybridisations}

Four independent labelling (including dye-swaps) reactions per species were carried out using $25 \mu \mathrm{g}$ total RNA from both human and bovine per labelling reaction. Direct incorporation of Cy3 and Cy5 during reverse transcription was carried out in a $20 \mu \mathrm{l}$ reaction volume using $1 \mu \mathrm{g}$ of anchored oligo-dT primer. The RNA/primer mix was incubated at $70^{\circ} \mathrm{C}$ for $5 \mathrm{~min}$., left at room temperature for $10 \mathrm{~min}$ and then cooled on ice for $2 \mathrm{~min}$. The following reagents were then added: $4 \mu \mathrm{l}$ first-strand buffer, $2 \mu \mathrm{l}$ $0.1 \mathrm{M}$ DTT, $0.5 \mu \mathrm{l}$ dNTP mix ( $25 \mathrm{mM}$ each of dATP, dGTP, dCTP and $10 \mathrm{mM}$ dTTP), $1 \mu \mathrm{l}$ of $1.0 \mathrm{mM}$ Cy5- or Cy3dUTP (Amersham Pharmacia) and $1 \mu \mathrm{l}(200 \mathrm{U} / \mu \mathrm{l})$ Superscript II (Invitrogen). The labelling reaction was carried out at $42^{\circ} \mathrm{C}$ for $1.5 \mathrm{hrs}$. The reaction was stopped with $4 \mu \mathrm{l}$ of $0.5 \mathrm{M}$ EDTA. The input RNA was hydrolysed by the addition of $2 \mu \mathrm{l}$ of $2.5 \mathrm{M} \mathrm{NaOH}$ and incubated at $37^{\circ} \mathrm{C}$ for 15 mins. followed by neutralization with $10 \mu \mathrm{l}$ HEPES free acid ( $2 \mathrm{M}, \mathrm{pH}$ 5.5). Labelled cDNAs (four replicates per species which included dye-swaps) were purified from unincorporated Cy-dyes using Microcon YM-30 purification columns (Millipore). All labelled cDNAs were routinely analysed on a Fuji scanner (FL8-8000) and by ethidium bromide staining to ascertain dye incorporation and size-range of synthesized cDNAs. After concentrating cDNAs by evaporation in a SpeedVac, labelled targets were resuspended in $20 \mu \mathrm{l}$ of hybridisation buffer $(10 \mu \mathrm{g}$ polydA and $20 \mu \mathrm{g}$ Human Cot1 DNA,-Invitrogen; DIGEasy Hybridisation mix -Roche). After thorough resuspension, the cDNA was denatured by heating at $95^{\circ} \mathrm{C}$ for 5 mins followed by 20 mins. at $42^{\circ} \mathrm{C}$ to enable annealing of the blocking reagents to repetitive sequences within the target cDNAs. The hybridisation mixture consisting of Cy3-labelled bovine and Cy5-labelled human RNA and vice versa was placed on the blocked array under a $24 \times 40$ mm coverslip (Menzel-Glaser, Germany). To maintain humidity inside the chamber, $20 \mu \mathrm{l}$ of 3x SSC was added to the reservoir wells. The chamber was then tightly sealed and slides incubated at $42^{\circ} \mathrm{C}$ for $18 \mathrm{hrs}$ in a waterbath. Slides were washed twice in $0.2 x$ SSC / $0.1 \%$ SDS and then twice in $0.2 \mathrm{x}$ SSC. Washes were carried out at room temperature with $10 \mathrm{~min}$ durations per wash. Finally, the slides were dried by centrifugation at $1100 \mathrm{rpm}$ for 10 $\min$.

\section{Image acquisition and data analysis}

Fluorescence images were captured using an Affymetrix 428 scanner (Affymetrix, Santa Clara, CA) with appropriate gains on the photomultiplier tube (PMT) to obtain the highest intensity without saturation. A 16 bit TIFF image was generated for each channel for subsequent image analyses. Image analysis was carried out by placing the centre of each spot manually (grid-finding step) using the software AIDA (Raytest-Germany) and then by quantifying in a pre-defined neighbourhood around this spot centre using a two-dimensional Gaussian distribution (quantification step).

Data analysis comprised two distinct parts. In the first part, data were normalised to eliminate extrinsic influencing factors and artefacts not attributable to the probe-target interaction. For each tissue (human and bovine brain) under analysis the whole batch of experimental replicates was normalized simultaneously. Normalisation should eliminate multiplicative technical bias between the different experiments and result in the same median signal level for each experiment. In a first step, the local background of each spot was subtracted from the spot's signal intensity. Then, for each experiment $j$ the median signal, med $_{j^{\prime}}$ was computed and a multiplicative factor was calculated according to $\mathrm{med}_{\mathrm{ref}} / \mathrm{med}_{\mathrm{j}}$, where $\operatorname{med}_{\mathrm{ref}}$ is the global derived from the average intensity values of the cDNAs across the full batch of experiments. The multiplicative factor was used to adjust the signal of the ith cDNA in the $j$ th experiment, $\mathrm{x}_{\mathrm{ij}}$, by $\mathrm{x}_{\mathrm{ij}}$ * $\mathrm{med}_{\mathrm{ref}} / \mathrm{med}_{\mathrm{j}}$.

In the second part, a number of numerical characteristics were calculated in order to quantify the cross-species comparison. These characteristics were signal detection value, signal reproducibility and statistical significance of differential expression. Signal detection was judged by a number of empty positions that were spread across the array. For each experiment, the proportion of signals of empty positions lower than the actual spot signal was calculated. Then across all repetitions, the average proportion was kept as the signal detection value. Employing this procedure, a signal strength was quantified for each cDNA. Signal reproducibility was judged by calculating 
the co-efficient of variation (CV) for each cDNA across all experiments.

In order to judge differential expression of a gene in human and bovine brain we calculated three statistical tests: Student's t-test, Welch-test and Wilcoxon's rank sum test for each cDNA based on the signal series derived from human and bovine experimental repetitions. The three tests make different assumptions on the signal series. Whereas the first two assume that the series are Gaussian distributed, the Wilcoxon test is distribution-free. Low Pvalues calculated by these tests indicate significant differences in signal intensity within the human and bovine samples [15].

\section{Semi-quantitative RT-PCR analysis}

The list of primers, annealing temperatures and genes under investigation is shown in Table 1.

For primer annealing and reverse transcription, $1.0 \mu \mathrm{l}$ $(2.5 \mu \mathrm{g} / \mu \mathrm{l})$ of DNase1 treated human and bovine total RNA was added to $2.0 \mu \mathrm{l}(50 \mu \mathrm{M})$ Oligo-dT primer plus $9.0 \mu \mathrm{l}$ of RNAse free water. The mixture was spun briefly and heated to $70^{\circ} \mathrm{C}$ for 5 mins and cooled on ice. Thereafter, the following components were added sequentially, $4.0 \mu \mathrm{l}$ of $5 \mathrm{x}$ RT. Buffer (Invitrogen), $2.0 \mu \mathrm{l}$ of $0.1 \mathrm{M}$ DTT (Invitrogen) $1.0 \mu \mathrm{l}$ of $(10 \mathrm{mM}) \mathrm{dNTP}$ (Amersham) and $1.0 \mu \mathrm{l}(200 \mathrm{U} / \mu \mathrm{l})$ Superscript II (Invitrogen). After pulse spinning, incubation was carried out at $42^{\circ} \mathrm{C}$ for $1.5 \mathrm{hrs}$.

Gene-specific PCR amplifications were carried out in a total volume of $50 \mu \mathrm{l}$ consisting of $5 \mu \mathrm{l}$ of $10 \mathrm{x}$ PCR buffer, $0.2 \mu \mathrm{l}(5 \mathrm{U} / \mu \mathrm{l}) \mathrm{Taq}$ Polymerase, $1.0 \mu \mathrm{l}(10 \mathrm{mM}) \mathrm{dNTP}, 2.5$ $\mu \mathrm{l}$ of each $(20 \mu \mathrm{M})$ primer, $1.5 \mu \mathrm{l}(50 \mathrm{mM}) \mathrm{MgCl}_{2}, 2.0 \mu \mathrm{l}$ of the first strand CDNA (equivalent to $125 \mathrm{ng}$ of input RNA) and distilled water to $50 \mu \mathrm{l}$. All reagents were purchased from Invitrogen. Cycling parameters consisted of an initial denaturation step at $95^{\circ} \mathrm{C}$ for $5 \mathrm{~min}$ followed by 30 cycles of denaturation at $95^{\circ} \mathrm{C}$ for $30 \mathrm{sec}$, annealing for $30 \mathrm{sec}$, elongation at $72^{\circ} \mathrm{C}$ for $30 \mathrm{sec}$, then a final elongation step at $72^{\circ} \mathrm{C}$ for $5 \mathrm{~min}$. Primer sequences, annealing temperatures and predicted size of cDNA products are shown in Table 1 . The amplification reaction was carried out in a PTC 200 PCR machine (MJ Research). After PCR amplification, $50 \mu \mathrm{l}$ of the reaction products were resolved on 2.0 percent agarose gel containing $0.2 \mu \mathrm{g} / \mathrm{ml}$ ethidium bromide. The gel was placed on a U.V. transilluminator (UVP Model TFM-20, Ultra-Violet Products Ltd., Cambridge, U.K.) with 25-watt UV tubes for high fluorescence and high sensitivity on stained gels), and imaged with a Photometrics Quantix 1401E, 12-bit cooled CCD camera (Roper Scientific GmbH, Ottobrunn, Germany). Densitometric analysis of the digitized image was performed with the IPLab-Gel program from Scanalytics (Fairfax, VA, U.S.A.). The semi-quantitative RT-PCR assay provides sensitive and reliable results, (26). The linear range of amplification was determined by product quantification after different cycle numbers. Identical amounts of bovine and human mRNA were amplified with the same number of PCR cycles and were always placed next to each other on the gel. Each RT-PCR was repeated at least three times for each gene. The housekeeping gene $\beta$ ACTIN was used as endogenous control.

\section{Authors' contribution}

J.A. conceived the study's idea, designed and optimised the protocols, carried out the hybridisations and was pivotal in developing the analysis plan and writing the manuscript.

R.H. developed the analysis plan, analysed the data and was pivotal in writing the manuscript.

D.H and M.N designed the cross-species primers and carried out the semi-quantitative RT-PCR.

W.W. performed the initial image analysis.

A.BK. selected the clones used as probes for creating the Chip and carried out the assignment of orthologous genes.

T.B. assisted in the semi-quantitative RT-PCR analysis

J.W.C. was involved in the conceptualisation and writing

C.H. was instrumental in the Chip design and production.

H.N. is the Head of the Department of Biotechnology at the Institute for Animal Science and was involved in the conceptualisation and writing.

H.L. is the Head of the Department of Vertebrate Genomics at the Max Planck Institute for Molecular Genetics.

\section{Additional material}

Additional File 1 


Summary of the expression data and the corresponding gene annota-
tions. The terms used under each column are explained viz;IMAGE ID:
Gene specific identifier assigned by the IMAGE consortiumGene: HUGO
gene nameChromosome: Chromosomal location of geneDescription: Gene
descriptionMolecular Function: Assigned function by the Gene Ontology
ConsortiumBG-tag-bovine: Signal detection probability in the bovine sam-
plesBG-tag-human: Signal detection probability in the human samp-
lesCV-bovine: Co-efficient of variation between replicate signal
intensitiesCV-human: Co-efficient of variation between replicate signal
intensitiesMean-bovine: Mean signal across all hybridisation experiments
using bovine RNAlog-Mean-bovine: Logarithm (base 2) of the mean val-
ueMean-human: Mean signal across all hybridisation experiments using
human RNAlog-Mean-human: Logarithm (base 2) of the mean valueR-
atio(bovine vs human): Ratio of the mean valueslog2(Ratio): Log-ratio
(base 2) of the mean valuesP-value-Student's-t-test: P-value of Student's
t-test using the replicate experimentsP-value-Welch-test: P-value of the
Welch test using the replicate experimentsP-value-Wilcoxon-test: P-value
of Wilcoxon's test using the replicate experimentsMatch: A BLAST hit
with E-value <1.0e-15 was found (1) or not (0)Homology: Classification
of sequence homology between human gene sequence and bovine ESTs.
"ortholog" = best match in both directions, "paralog" = bovine EST has its
best match with another human sequence.TIGR-BTGIO-51503-Matches:
Best results of the BLAST matches using the Ensembl- annotated gene
sequence with the TIGR- databaseIdentity: \%-identity of best matchOver-
lap: Overlap in base pairs
Click here for file
[http://www.biomedcentral.com/content/supplementary/1471-
$2164-5-83-S 1 . x l s]$

\section{Acknowledgements}

We are greatful to Dr Marie-Laure Yaspo for reading the manuscript and providing valuable comments and suggestions, the microarray support staff and the RZPD for clone picking and sequencing. This work was funded by the German Ministry for Education and Research (BMBF) as part of the National Genome Research Network (NGFN).

\section{References}

I. Schena M, Shalon D, Davis RW, Brown PO: Quantitative monitoring of gene expression patterns with a complementary DNA microarray. Science 1995, 270:467-70.

2. Chismar JD, Mondala T, Fox HS, Roberts E, Langford D, Masliah E, Salomon DR, Head SR: Analysis of result variability from highdensity oligonucleotide arrays comparing same-species and cross-species hybridisations. BioTechniques 2002, 33:516-524.

3. Fisslthaler B, Popp R, Kiss L, Potente M, Harder DR, Fleming I, Busse $\mathrm{R}$ : Cytochrome P450 2C is an EDHF synthase in coronary arteries. Nature 1999, 40 I:493-497.

4. Moody DE, Zou Z, Mclntyre L: Cross-species hybridisation of pig RNA to human nylon microarrays. BMC Genomics 2002, 3:27.

5. Medhora M, Bousamra M, Zhu D, Somberg L, Jacobs ER: Upregulation of collagens detected by gene array in a model of flowinduced pulmonary vascular remodeling. Am J Physiol Heart Circ Physiol 2002, 282: $\mathrm{H} 414-22$.

6. Huang GS, Yang SM, Hong MY, Yang PC, Liu YC: Differential gene expression of livers from ApoE deficient mice. Life Sciences 2000, 68: 19-28.

7. Tsoi SC, Cale JM, Bird IM, Ewart V, Brown LL, Douglas S: Use of human cDNA microarrays for identification of differentially expressed genes in Atlantic Salmon liver during Aeromonas salmonicida infection. Mar Biotechnol (NY) 2003, 5:545-54.

8. Niemann H, Wrenzycki C: Alterations of expression of developmentally important genes in preimplantation bovine embryos by in vitro culture conditions: Implications for subsequent development. Theriogenology 2000, 53:2I-34.

9. Lazzari L, Wrenzycki C, Herrmann D, Duchi R, Kruip T, Niemann H, Galli C: Cellular and molecular deviations in bovine in vitro produce embryos are related to the Large Offspring Syndrome. Biol Reprod 2002, 67:767-775.

10. Adjaye J, Bolton V, Monk M: Developmental expression of specific genes detected in high quality cDNA libraries from single human preimplantation embryos. Gene 1999, 237:373-383.

II. Brambrink T, Wabnitz P, Halter R, Klocke R, Carnwath J, Kues W, Wrenzycki C, Paul D, Niemann D: Application of cDNA arrays to monitor mRNA profiles in single preimplantation mouse embryos. Biotechniques 2002, 33:376-385.

12. Goto T, Adjaye J, Rodeck C, Monk M: Identification of human primordial germ cell specific transcribed genes by differential display. Molecular Human Reproduction 1999, 5(9):85I-860.

13. Pan GJ, Chang ZY, Schöler H, Duanqing PE: Stem cell pluripotency and transcription factor Oct4. Cell Research 2002, I 2(56):32I-329.

14. Yang YH, Dudoit S, Luu P, Lin DM, Peng V, Ngai J, Speed TP: Normalization for cDNA microarray data: A robust composite method addressing single and multiple slide systematic variation. Nucleic Acids Res 2002, 30(4): el 5.

15. Kahlem P, Sultan M, Herwig R, Steinfath M, Balzereit D, Eppens B, Saran NG, Pletcher MT, South ST, Stetten G, Lehrach H, Reeves RH, Yaspo ML: Transcript level alterations reflect gene dosage effects across several tissues in a mouse model of Down Syndrome. Genome Res 2004, 14:1258-1267.

16. Herwig R, Aanstad P, Clark M, Lehrach H: Statistical evaluation of differential expression on cDNA nylon arrays with replicated experiments. Nucleic Acids Res 200I, 29(23): I I 7.

17. Storey JD, Tibshirani R: Statistical significance for genomewide studies. Proc Natl Acad Sci USA 2003, 100:9440-9445.

18. Frazer KA, Elnitski L, Church DM, Dubchak I, Hardison RC: Crossspecies sequence comparison: $A$ review of methods and available resources. Genome Res 2003, I3(I): I-I2.

19. Evertsz EM, Au-Young J, Ruvolo MV, Lim AC, Reynolds MA: Hybridization cross-reactivity within orthologous gene families on glass microarrays. BioTechniques 200 I, 31 I:I I82-1 I 92.

20. Miller NA, Gong Q, Bryan R, Ruvolo M, Turner LA, LaBrie ST: Crosshybridization of closely related genes on high-density macroarrays. BioTechniques 2002, 32:620-625.

21. Jimenez JL, Mitchell MJ, Sgouros JG: Microarray analysis of orthologous genes:conservation of the translational machinery across species at the sequence and expression level. Genome Biology 2002, 4:R4.

22. Mastrangelo T, Modena P, Tornielli S, Bullrich F, Testi MA, Mezzelani A, Radice P, Azzarelli A, Pilotti S, Croce CM, Pierotti MA, Sozzi G: A novel zinc finger gene is fused to EWS in small round cell tumor. Oncogene 2000, 19(33):3799-3804.

23. Makalowski W, Zhang W], Boguski M: Comparative analysis of 1196 orthologous mouse and human full-length mRNA and protein sequences. Genome Res 1996, 6:846-857.

24. Girke T, Todd J, Ruuska S, White J, Benning C, Ohlrogge J: Microarray analysis of developing Arabidopsis seeds. Plant Physiol 2000, 124:1570-I58I.

25. Kane MD, Jatkoe TA, Stumpf CR, Lu J, Thomas JD, Madore SJ: Assesment of the sensitivity and specificity of oligonucleotide (50 mer) microarrays. Nucleic Acids Res 2000, 28:4552-4557.

26. Diehl F, Beckmann B, Kellner N, Hauser NC, Diehl S, Hoheisel JD: Manufacturing DNA microarrays from unpurified PCR products. Nucleic Acids Res 2002, 30:e79.

27. Knijn HM, Wrenzycki C, Hendriksen PJM, Vos PLAM, Herrmann D, van der Weijden GC, Niemann H, Dieleman SJ: Effects of oocyte maturation regimen on the relative abundance of gene transcripts in bovine blastocysts derived in vitro or in vivo. Reproduction 2002, 124:365-375. 